\title{
A potential role for protein palmitoylation and zDHHC16 in DNA damage response
}

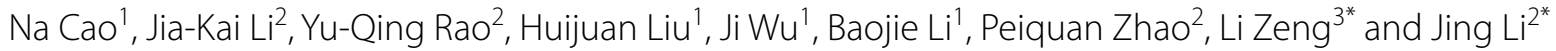

\begin{abstract}
Background: Cells respond to DNA damage by activating the phosphatidylinositol-3 kinase-related kinases, p53 and other pathways to promote cell cycle arrest, apoptosis, and/or DNA repair. Here we report that protein palmitoylation, a modification carried out by protein acyltransferases with zinc-finger and Asp-His-His-Cys domains (zDHHC), is required for proper DNA damage responses.

Results: Inhibition of protein palmitoylation compromised DNA damage-induced activation of Atm, induction and activation of p53, cell cycle arrest at G2/M phase, and DNA damage foci assembly/disassembly in primary mouse embryonic fibroblasts. Furthermore, knockout of zDHHC16, a palmitoyltransferase gene identified as an interacting protein for c-Abl, a non-receptor tyrosine kinase involved in DNA damage response, reproduced most of the defects in DNA damage responses produced by the inhibition of protein palmitoylation.
\end{abstract}

Conclusions: Our results revealed critical roles for protein palmitoylation and palmitoyltransferase zDHHC16 in early stages of DNA damage responses and in the regulation of Atm activation.

Keywords: Protein palmitoylation, DNA damage response, zDHHC16

\section{Background}

Protein palmitoylation, or protein S-acylation, is a posttranslational modification that adds a palmitate moiety to specific Cys residues by a family of proteins named protein acyltransferases (PATs) [1-4]. All PAT proteins contain a DHHC domain, a 51-amino acid Cys-rich domain with a highly conserved Asp-His-His-Cys sequence. The other regions of the PATs are variable. The $\mathrm{zDHHC}$ genes are numerically named zDHHC1-24 [4]. Unlike N-myristoylation or C-prenylation, $\mathrm{S}$-acylation can be reversed by protein palmitoyl thioesterases and acyl protein thioesterases, making it a reversible lipid modification [5].

A number of cellular proteins have been reported to be palmitoylated, which are involved in different cellular

\footnotetext{
*Correspondence: li_zeng@nni.com.sg; lj_xinhua@126.com

2 Department of Ophthalmology, Xin Hua Hospital, Shanghai Jiao Tong University School of Medicine, Shanghai, China

${ }^{3}$ Neural Stem Cell Research Lab, Research Department, National

Neuroscience Institute, Singapore 308433, Singapore

Full list of author information is available at the end of the article
}

activities such as cell signaling, protein trafficking, and cell adhesion [6-8]. Most of the palmitoylated proteins are membrane or peripheral membrane proteins, yet it is noteworthy that some non-membrane associated proteins are also palmitoylated. The importance of protein palmitoylation is manifested in $\mathrm{zDHHC}$ deficient mouse models. For example, mice deficient for zDHHC8 gene have increased risk of schizophrenia [9]. Mice with zDHHC13 mutation show alopecia, osteoporosis, and amyloidosis [10]. A spontaneous mutation of $\mathrm{zDHHC} 21$ gene in mice leads to hair loss due to defective epidermal homeostasis and hair follicle differentiation [11]. We have recently reported that mice null of zDHHC16 gene are neonatal lethal with severe heart and eye defects [12].

Recent studies have also implicated protein palmitoylation and PATs in cancer development. The expression of some zDHHC genes was found altered in various cancer tissues [13, 14]. Yet how PATs participate in cancer development remains unclear. Cancer development is driven by the accumulation of gene mutations, especially 
loss-of-function mutations of tumor suppressors and gain-of-function mutations of oncoproteins [15]. However, cell has a protective system, the DNA damage response (DDR), to monitor DNA damage and to repair the damage or eliminate the cells with irreparable DNA lesions [16-18]. Upon DNA damage, the cell activates the phosphatidylinositol-3 kinase-related kinases (PIKKs) such as Atm and Atr at the DNA break sites. A large number of proteins, including $\gamma \mathrm{H} 2 \mathrm{AX}$ and BRCT domain-containing proteins such as Brca1, TopBP1, and Mdc1 are recruited to the DNA break sites, forming transient nuclear structures named DNA damage foci, which are thought to be the centers for signal propagation and DNA repair [19-21]. Atm phosphorylates many substrates including Smad1, p53 and Chk2, which eventually cause cell cycle arrest and/or apoptosis [22-25]. Thus, a functional DDR is critical for maintaining genome integrity and preventing tumor development. On the other hand, tumor cells usually have disrupted DDR [18]. Up to date, it is not known whether protein palmitoylation plays a role in DNA damage response.

In the present study, we investigated the roles of protein palmitoylation in DNA damage response. We found defective DDR including Atm activation, p53 induction and activation, cell cycle arrest at G2/M phase, and assembly/disassembly of DNA damage foci in primary mouse embryonic fibroblasts (MEFs) in the presence of 2-bromopalmitate (2BP), a general PAT inhibitor [26-28]. These results were also observed in MEFs deficient of $z D H H C 16$ gene which encodes a palmitoyltransferase. These findings, for the first time, unravel an important function of PATs, in particular zDHHC16, in DNA damage response and in Atm activation, and provide a possible explanation on how $\mathrm{zDHHC}$ proteins participate in tumorigenesis.

\section{Methods}

\section{Mice and cells}

Mice were housed, bred and used in a specific pathogen free (SPF) animal facility at the Bio-X Institute, Shanghai Jiao Tong University. Specifically, no more than five adult mice were housed in one individually ventilated cage with sterilized food, water and woodchip bedding. The animal facility was maintained by professional care

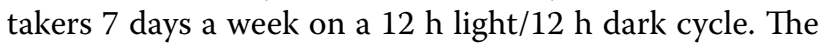
study was approved by the Institutional Animal Care and Use Committee of Shanghai Jiao Tong University [SYXK(SH)2011-0112]. Timed pregnant female mice were euthanized on embryonic E13.5 by intraperitoneal injection of over-dosed pentobarbital. The time of pregnancy was determined by visual examination of the vaginal plug in the early morning. Embryos were dissected and fibroblasts were isolated as described previously [24]. The generation and characterization of the zDHHC16 knockout mice were described in detail in our previous paper [12]. One pregnant $\mathrm{C} 57 \mathrm{Bl} / 6$ wildtype and three zDHHC16 knockout mice were used to obtain all MEFs used in this study. The knockout mice were in mixed C57BL/6 and CBA background. All efforts were made to minimize the suffering of mice.

Cells were cultured in Dulbecco's modified Eagle's medium (Thermo Fisher Scientific Inc./Life Technologies, Grand island, NY, USA) containing $10 \%$ fetal calf serum (Excell Biology Inc., Shanghai, China). They were plated at $10^{6}$ cells per $6 \mathrm{~cm}$ dish and allowed to grow overnight before any treatment. To inhibit cellular PAT activity, 2BP (2-bromopalmitate, Sigma-Aldrich, China) was used at 50 or $100 \mu \mathrm{M}$ for $24 \mathrm{~h}$ as indicated. To induce DNA damage response, doxorubicin (Dox) (Selleck Chemicals, Houston, TX, USA) was used at $1 \mu \mathrm{M}$ for different time as indicated in each experiment.

\section{Western blot analysis}

Standard RIPA buffer containing $1 \mathrm{mM}$ PMSF, $1 \mu \mathrm{g} / \mathrm{mL}$ aprotonin, leupeptin, and pepstatin was used for protein extraction. Protein concentration was measured using Bio-Rad DC protein assay kit (Bio-Rad Inc., Hercules, CA, USA). Western blot analysis was carried out according to the standard procedure. We used polyvinylidene fluoride membrane for protein transfer, and $5 \%$ nonfat dried milk in PBS as the blocking agent. All primary antibodies were incubated overnight at $4{ }^{\circ} \mathrm{C}$. Chemiluminescent detection method (ECL kit, GE Healthcare, Buckinghamshire, UK) coupled with Bio-Rad ChemiDoc XRS imaging system were used for the detection, visualization and quantitation of the proteins. All primary antibodies were purchased from cell signaling technology and used according to the provider's instruction except for the following: anti-Atm antibody was purchased from ECM Biosciences (AM3611), anti-p-Atm antibody was purchased from Millipore (05-740) and anti- $\beta$-Actin was purchased from Santa Cruz (SC81178).

\section{Flow cytometry}

Cells were digested with $0.25 \%$ trypsin, washed with cold PBS and fixed in $70 \%$ ethanol at $-20{ }^{\circ} \mathrm{C}$ overnight. At the next day, cells were washed with cold PBS again and incubated in PBS containing $50 \mu \mathrm{g} / \mathrm{mL}$ propidium iodide (PI) and $100 \mu \mathrm{g} / \mathrm{mL}$ RNase A for $40 \mathrm{~min}$ in the dark at room temperature. The fixed and labeled cells were analyzed with Becton-Dickinson FACSCalibur (BD Bioscience, San Jose, CA, USA).

\section{In vitro analysis of DNA damage foci positive for $\mathrm{\gamma H} 2 \mathrm{AX}$, TopBP1, and BRCA1}

Cells cultured on glass slides were fixed in $4 \%$ paraformaldehyde/PBS for $30 \mathrm{~min}$ followed by $0.1 \%$ 
TritonX-100/PBS incubation for $40 \mathrm{~min}$ at room temperature. The standard immunostaining procedure was used. Specifically, $10 \%$ goat serum was used as the blocking agent. Primary antibody incubation was carried at $4{ }^{\circ} \mathrm{C}$ overnight. Anti-p-H2AX antibody was purchased from Millipore (05-636), anti-TopBP1 antibody was purchased from BD Bioscience (611875), and antiBrca1 antibody was purchased from Abcam (ab191042).

\section{Reverse transcription (RT)-polymerase chain reaction (PCR)} Trizol reagent (Invitrogen, Thermo Fisher Scientific, Grand island, NY, USA) was used to extract whole RNA and reverse transcribed using SuperScript III reverse transcriptase and random primer (Invitrogen, ThermoFisher Scientific, USA). Relative quantitative PCR was performed using the primers listed in Table 1 and the FastStart Universal SYBR Green Master from Roche (Roche Diagnostic $\mathrm{GmbH}$, Mannheim, Germany). $\beta$-actin and glyceraldehyde 3 -phosphate (GAPDH) were used as internal controls.

\section{Statistical analysis}

Each experiment was repeated at least three times or using cells isolated from three mutant and control mice. The results were analyzed using analysis of variance (ANOVA) with Fisher's LSD (least significant difference) test when more than two groups were compared or unpaired $t$ test when two groups were compared (SPSS version 18). p Value of equal or less than 0.05 was accepted as statistically significant.

\section{Results}

\section{Most of the zDHHC genes are expressed in MEFs}

We chose to use primary MEFs for this study as most of the immortal cell lines show disrupted DNA damage response [18]. We first wanted to document which of the 23 protein-coding $\mathrm{zDHHC}$ genes were expressed in MEFs and whether their expression was altered by DNA damage. Relative quantitative PCR analysis showed that MEFs expressed $20 \mathrm{zDHHC}$ genes (Fig. 1). The mRNA for zDHHC19, 22 and 23 was not detectable in MEFs. However, they were found in mouse retina tissues (data not shown). We then treated MEFs with Dox, a chemotherapeutic drug that generates double-stranded and single-stranded DNA breaks [29], and analyzed the mRNA levels of the zDHHC genes. Only zDHHC11, 17, 20 and 24 mRNA showed increase of about twofold, while the rest exhibited no significant change. The expression of a wide spectrum of PATs in primary MEFs suggests that protein palmitoylation may play important roles in these cells.

\section{Impaired DNA damage-induced p53 activation in the presence of 2BP}

We then wanted to study the possible roles of protein palmitoylation in DNA damage response. Since it was unfeasible to simultaneously silence most of the PATs with interference RNA, we used 2BP, a substrate analog inhibitor that had been widely used to block PAT activity [30-32]. Although it may have activities other than palmitoylation inhibition [26], we have shown that 2BP at the concentrations of $50 \mu \mathrm{M}$ and above was able to inhibit total PAT activities in cultured cells [12, 33]. Indeed, reduced protein palmitoylation was observed in MEFs in the presence of $50 \mu \mathrm{M} 2 \mathrm{BP}$ (Additional file 1: Figure S1). Additionally, 2BP showed very modest effects on the expression of a few $\mathrm{zDHHC}$ genes (Additional file 1: Figure S2).

Western blot analysis showed that Dox-induced increase in the protein levels of p53, phosphorylation of p53 at Ser15, and the protein levels of p21 and Bax, targets of p53, were inhibited in the presence of 2BP (Fig. 2a). While Dox induced a near fivefold increase of phosphorylated p53 in normal MEFs by $24 \mathrm{~h}$, the increase was only 2.5 -fold or none in cells with 50 and $100 \mu \mathrm{M}$ 2BP pre-treatment, respectively.

To further confirm these findings, we analyzed mRNA levels of p21 (Cdkn1a), Bax, and Puma (Bbc3) using relative quantitative PCR. We found that Dox treatment increased the mRNA levels of p21 and Bax but not Puma in MEFs (Fig. 2b). 2BP significantly inhibited the induction of $\mathrm{p} 21$ and Bax expression at the mRNA levels in response to Dox treatment (Fig. 2b). Since one of the main functions of 2BP was to inhibit PATs, these results suggest that protein palmitoylation is required for the optimal induction of p53 target genes.

\section{Impaired DNA damage-induced Atm activation in the presence of 2BP}

The induction and activation of p53 is dependent on Atm activation in response to double-stranded DNA breaks. We found decreased activation of Atm in primary MEFs, justified by decreased Atm phosphorylation on Ser1981 (Fig. 3a, b) in the presence of 2BP. Although Ser1981 phosphorylation is not required for Atm activation in vivo, it is an autophosphorylation commonly used as an indication of Atm activation [22]. Moreover, we found that 2BP also impeded DNA damage-induced Smad1 activation (Fig. 3c), another cellular event that requires Atm activation [24]. 
Table 1 The primer sequences used in relative quantitative PCR

\begin{tabular}{|c|c|c|c|c|}
\hline Refseq gene & Accession number & Forward primer $\left(5^{\prime}-3^{\prime}\right)$ & Reverse primer $\left(5^{\prime}-3^{\prime}\right)$ & Amplicon size \\
\hline Gapdh & NM_008084 & TGACCTCAACTACATGGTCTACA & CTTCCCATTCTCGGCCTTG & 85 \\
\hline Actb & NM_007393 & GGCTGTATTCCCCTCCATCG & CCAGTTGGTAACAATGCCATGT & 154 \\
\hline Zdhhc1 & NM_175160 & ATGAACATCTGCAACAAACCCT & GCTCCATCCATTCCTTCGAGAG & 126 \\
\hline Zdhhc2 & NM_178395 & TGCTGGGCTGGTCCTACTAC & TGATAAGCCATGAGGCACAA & 94 \\
\hline Zdhhc3 & NM_178395 & ATCCCCACCCATCACTTCC & CTCGGATAAACCACATGGCTC & 112 \\
\hline Zdhhc4 & NM_026917 & TTACCTAGATGACGTGGGGC & AAACGATGACAAAGCCCAGT & 110 \\
\hline Zdhhc5 & NM_028379 & CCGCCATATTTCTAGTGGGA & TTGCATTGTAAATGGGCACT & 99 \\
\hline Zdhhc6 & NM_144887 & GAGTAAGAGGGTGGTTTCCTAGA & GCTGGATCTGAGTCACCATCAC & 67 \\
\hline Zdhhc7 & NM_025883 & CACCAGGAGCCTCAGCACT & AGCATCATGGGAGCACTTGT & 110 \\
\hline Zdhhc8 & NM_133967 & GGTTGGTTCCAGCACACTCT & AGAGGAAGAGGATGCCATTG & 98 \\
\hline Zdhhc9 & NM_172151 & GGGCATCTTCTACCTGACCC & AGACAGCTGAACAGCCAGGT & 91 \\
\hline zdhhc11 & NM_027704 & CTGACACCAATGTCCGACTCA & CGCGGTAACCTCACACAGG & 116 \\
\hline Zdhhc12 & NM_025428 & GGGAATCACTCTGGTGCTCT & ССССTTGСTCTTCССАTT & 104 \\
\hline Zdhhc13 & NM_028031 & GAGGCGTGCTTGGAGAGC & CTGGAACGTGGGAGCCAT & 129 \\
\hline Zdhhc14 & NM_146073 & CGGCGTCTTCTACCTGACTC & GATGGCAGGGGTGATCTTCT & 100 \\
\hline Zdhhc15 & NM_175358 & TGCCAGTGCTCGTTATTGTC & AACTTTTTCCGCTGGACTCA & 98 \\
\hline Zdhhc16 & NM_023740 & TACAGCTGCCAGCCTTTCC & CCCAACAGCAGACTTCGC & 115 \\
\hline zdhhc17 & NM_172554 & GCGGGAGGAGGGATTTAACAC & CCCGTTTCGGTCTCGTACTC & 63 \\
\hline zdhhc18 & NM_001017968 & TCAACGGGCAGACAGTGAAAC & GAAGCGGTAGTTCCGTCTCC & 158 \\
\hline Zdhhc19 & NM_199309 & TGTGACACTTGTGAAGGAACC & AAAAACAGCAGCAGCGTTACA & 106 \\
\hline Zdhhc20 & NM_029492 & ACCTTTGTGGTCGTCTGGTC & GCCACAAGGTAAACAACGGT & 104 \\
\hline Zdhhc21 & NM_026647 & GCTGCTTACTTGCTACGCAC & CTCATGGCGAACAACAAAGA & 100 \\
\hline Zdhhc22 & NM_001080943 & CGGCTGCTCAACGTGGTAG & CCAGGACGTAATTGCCCAGG & 190 \\
\hline Zdhhc23 & NM_001007460 & GGCTGCCTGTTTGTGTGATTG & CCGTGATTCTTTCGCAAGTCTC & 98 \\
\hline Zdhhc24 & NM_027476 & ACAGTGGCTCTCCTGCTGTT & CACACGTGTCCACCACAAA & 94 \\
\hline Zdhhc25 & NM_027306 & TTGGACTTACCTCGACCCAC & ATAGGGGCAGGTAGGGACAC & 109 \\
\hline Cdkn1a(p21) & NM_007669 & TCTCAGTGTTGAATACCGTGGG & TAAGGGTAGACAGTCCAGACCA & 115 \\
\hline Bax & NM_007527 & TGAAGACAGGGGCCTTTTTG & AATTCGCCGGAGACACTCG & 140 \\
\hline Bbc3(Puma) & NM_133234 & GCCCAGCCTGTAAGATACTGT & CCTTACAGGTAGTGCCAGATGC & 187 \\
\hline
\end{tabular}

The accession number was obtained from NCBI nucleotide sequence database

\section{Impaired DNA damage foci formation in the presence of 2BP}

We also looked at the formation of DNA damage foci in the presence of 2BP. In normal MEFs, Dox treatment induced DNA damage foci positive for $\gamma \mathrm{H} 2 \mathrm{AX}$, TopBP1, and Brca1, which are believed to be signal propagation centers as well as DNA repair centers (Fig. 4a, b). In 2BPtreated cells, the number of foci positive for $\gamma \mathrm{H} 2 \mathrm{AX}$ was higher than those in controls, especially at 8 and $16 \mathrm{~h}$ after Dox treatment. These results suggest that 2BP interferes with the assembly/disassembly of the DNA damage foci or the DNA repair process.

\section{Impaired DNA damage-induced cell cycle arrest in the presence of 2BP}

DNA damage eventually induces apoptosis or cell cycle arrest. Since 2BP compromised Atm activation and p53 induction and activation, we suspected that 2BP might affect Dox-induced cell cycle arrest and apoptosis. Cell cycle arrest was analyzed by flow cytometry (Fig. 5a). Dox treatment led to an accumulation of cells in the G2/M phase, a decrease in G1 phase cells, and a modest decrease in $\mathrm{S}$ phase cells in wild type MEFs. 2BP pre-treatment impeded Dox-induced increase in the percentage of cells arrested in the G2/M phase compared to control cells, without altering the percentage of $S$ phase cells (Fig. 5b). These results suggest that protein palmitoylation is required for proper $\mathrm{G} 2 / \mathrm{M}$ cell cycle arrest in DNA damage response. However, we found that 2BP showed some toxicity to MEFs at the doses used to inhibit protein palmitoylation, which may reflect a requirement for protein palmitoylation for cell survival, and the combination of 2BP and Dox caused more cell death (Additional file 1: Figure S3). This prevented us from further 


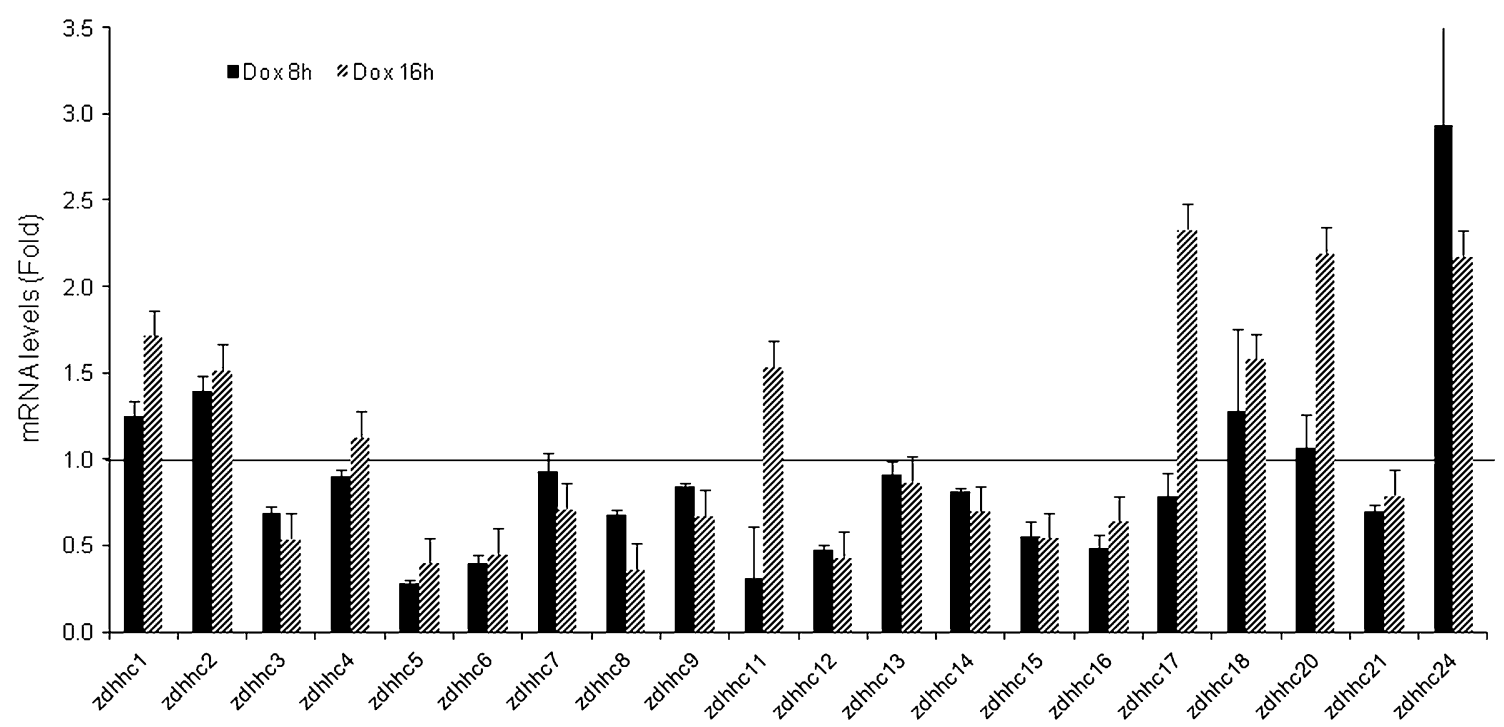

Fig. 1 ZDHHC gene expression in primary MEFs. The expression of each gene in primary MEFs without Dox treatment was set as 1 (as indicated by the horizontal line) and used to calculate the relative abundance of the same gene under Dox treatment for 8 and $16 \mathrm{~h}$ respectively. Gapdh and $\beta$-actin gene expression were used as internal controls and similar results were obtained. Error bars represented standard error means of repeated experiments. Notice that zDHHC10 is a pseudogene and the expression of zDHHC19, 22 and 23 genes were not detected

testing the role of 2BP-suppressed p53 activation in DNA damage-induced apoptosis.

\section{DHHC16 deficiency impaired the activation of Atm-p53}

We have previously reported the identification of one of the PATs, encoded by zDHHC16 gene. This protein, Aph2, was originally identified as a c-Abl interacting protein (Abl-philin2) [34]. c-Abl is involved in DNA damage response. In particular, it is required for Atm-p53 activation [35-38]. Biochemical and genetic studies have shown that at least one protein, phospholamban, a cardiac muscle specific protein, was palmitoylated by Aph2 [12]. In addition, MEFs overexpress zDHHC16 also showed increased total protein palmitoylation (Additional file 1: Figure S4a). Although Dox did not affect the expression of DHHC16 at the mRNA level (Additional file 1: Figure S2), Dox induced nuclear translocation of ectopically-expressed DHHC16 (Additional file 1: Figure S4b). We have tried to raise anti-DHHC16 antibodies but those antibodies could not recognize endogenous DHHC16. This could be due to the fact that DHHC16 is a membrane protein. The lack of anti-DHHC16 antibodies prevents us to study the expression, localization, and modification of endogenous DHHC16 at the moment. To test whether DHHC16 also plays a role in DDR, we treated primary MEFs deficient for zDHHC16 gene with Dox for different periods of time. Western blot analysis revealed that zDHHC16 deficiency, like inhibition of PATs with 2BP, compromised the activation of Atm and the induction of p53 and its target protein p21 (Fig. 6a-c).

\section{DHHC16 deficiency impaired DNA damage foci formation and cell death}

We next examined DNA damage foci formation in zDHHC16 deficient MEFs. Dox-treated zDHHC16 ${ }^{-1-}$ MEFs showed similar patterns of foci formation as the 2BP-treated wild type MEFs. The number of TopBP1 or Brcal positive foci in $\mathrm{zDHHC16} 6^{-1-} \mathrm{MEFs}$ was significantly higher than that in wild type cells (Fig. 7a-d). We also tested DNA damage-induced cell cycle arrest and apoptosis in $\mathrm{zDHHC} 16^{-/-}$and wild type MEFs. We found that Dox-induced G2/M cell cycle arrest in Aph $2^{-1-}$ MEFs were not significantly different from those in wild type MEFs (data not shown). However, zDHHC16 $6^{-/-}$MEFs showed a modest increase in cell survival compared to wild type MEFs (Fig. 7e). These results suggest that zDHHC16 plays a role in DNA damage-induced cell death.

\section{Discussion}

In this study, we showed that 2BP impaired Dox-induced DNA damage response, in particular the activation of the Atm-p53 pathway, and led to disrupted activation of the cell cycle checkpoint and DNA damage foci dynamics in primary MEFs. Since 2BP is a general PAT inhibitor which also binds palmitoylated proteins, we further showed that the defective DNA damage responses 

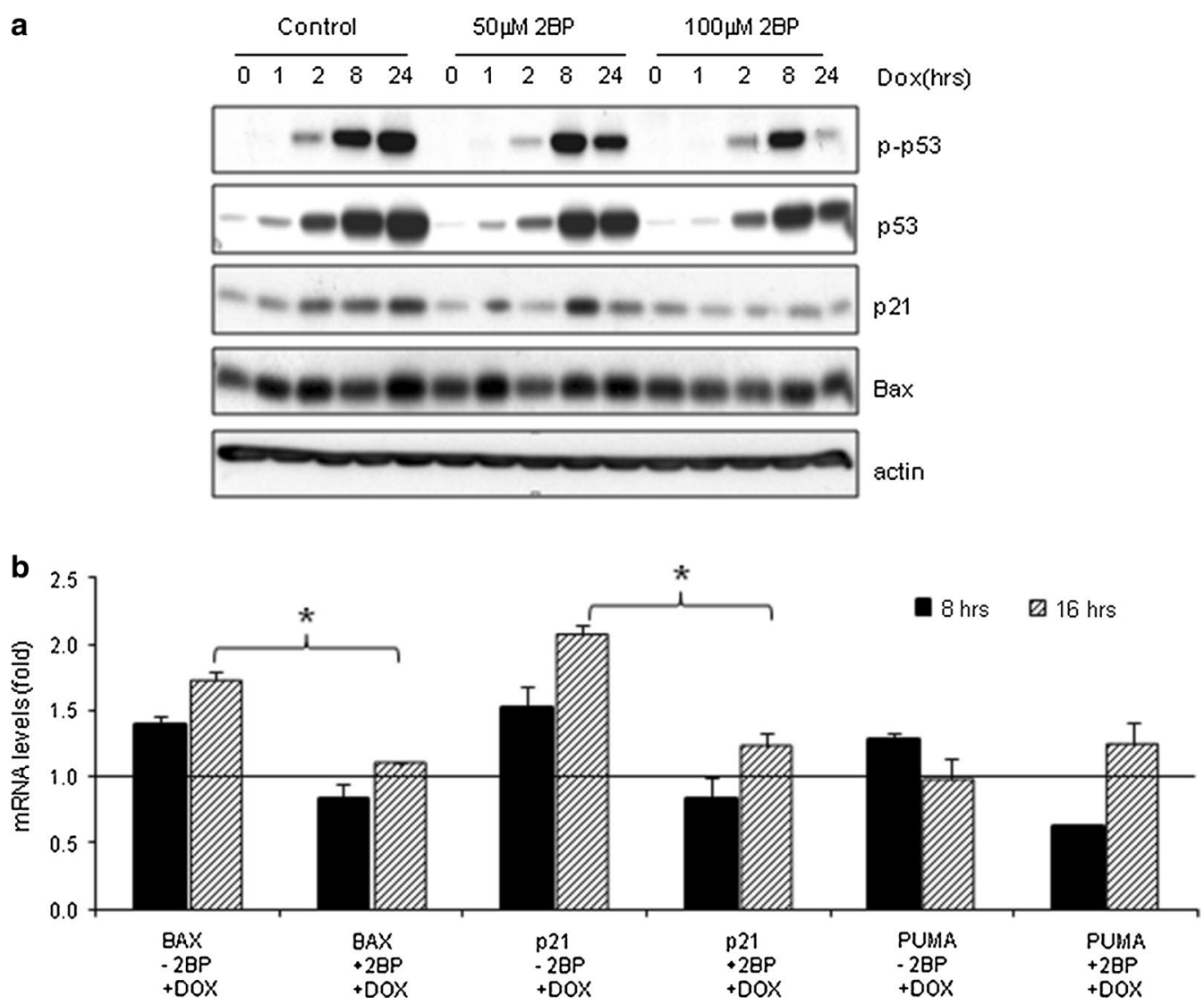

Fig. 2 Inhibition of palmitoylation by 2BP impaired DNA damage-induced p53 up-regulation and activation. a Western blot analysis showed that 2BP pre-treatment impeded the induction of p53 protein expression, p53 phosphorylation on Ser15, and p21 and Bax protein levels in response to Dox treatment. Primary MEFs were pre-treated with 50 or $100 \mu \mathrm{M} 2 \mathrm{BP}$ for $24 \mathrm{~h}$ and then stimulated with $1 \mu \mathrm{M}$ of Dox for different periods of time as indicated. The protein levels of p53, p-p53, p21, Bax, and actin were determined by Western blot analysis. b Relative quantitative PCR results showed that 2BP pre-treatment impeded the induction of Bax and p21 (Cdkn1a) gene expression, but not much of Puma (Bbc3) gene expression. The cells were pre-treated without (-2BP) or with $50 \mu \mathrm{M} 2 \mathrm{BP}(+2 \mathrm{BP})$ for $24 \mathrm{~h}$ and followed by $1 \mu \mathrm{M}$ Dox treatment for 0,8 and $16 \mathrm{~h}$. The expression of each gene without 2BP and Dox treatment was set as 1 (as indicated by the horizontal line) and used to compare the expression of the same gene under different stimulated conditions. Error bars represented standard error mean of the repeated experiments. Asterisk denoted significant difference $(p<0.05)$ etween Dox alone and 2BP plus Dox treated samples

observed in 2BP-treated cells were largely replicated in MEFs deficient for zDHHC16, one of the 23 palmitoyltransferases. Collectively our data suggested that protein palmitoylation carried out by PATs, in particular by zDHHC16, plays an important role in DNA damage response. Since DDR, in particular the Atm-p53 pathway, is the major tumor suppression scheme, our findings also provide a possible explanation on how some zDHHC proteins exert their tumor suppression activities [13].

Palmitoylation is a common protein post-translational modification $[1,3,14]$. This is also evident by the expression of a variety of $\mathrm{zDHHC}$ genes in primary MEFs.
However, little is known about the specific in vivo functions of each PAT. zDHHC16 knockout mice showed neonatal lethality with severe cardiac and ocular defects. It appears that one of the substrates of $\mathrm{zDHHC} 16$ protein in heart is phospholamban, which is largely responsible for cardiac defects observed in zDHHC16 knockout mice [12]. Here we found that cells deficient of zDHHC16 also had defective DNA damage response, a function that has not been previously ascribed to zDHHC16 or any other PATs.

How does zDHHC protein facilitate Atm activation? Since Atm is not known to be modified by palmitoylation, 

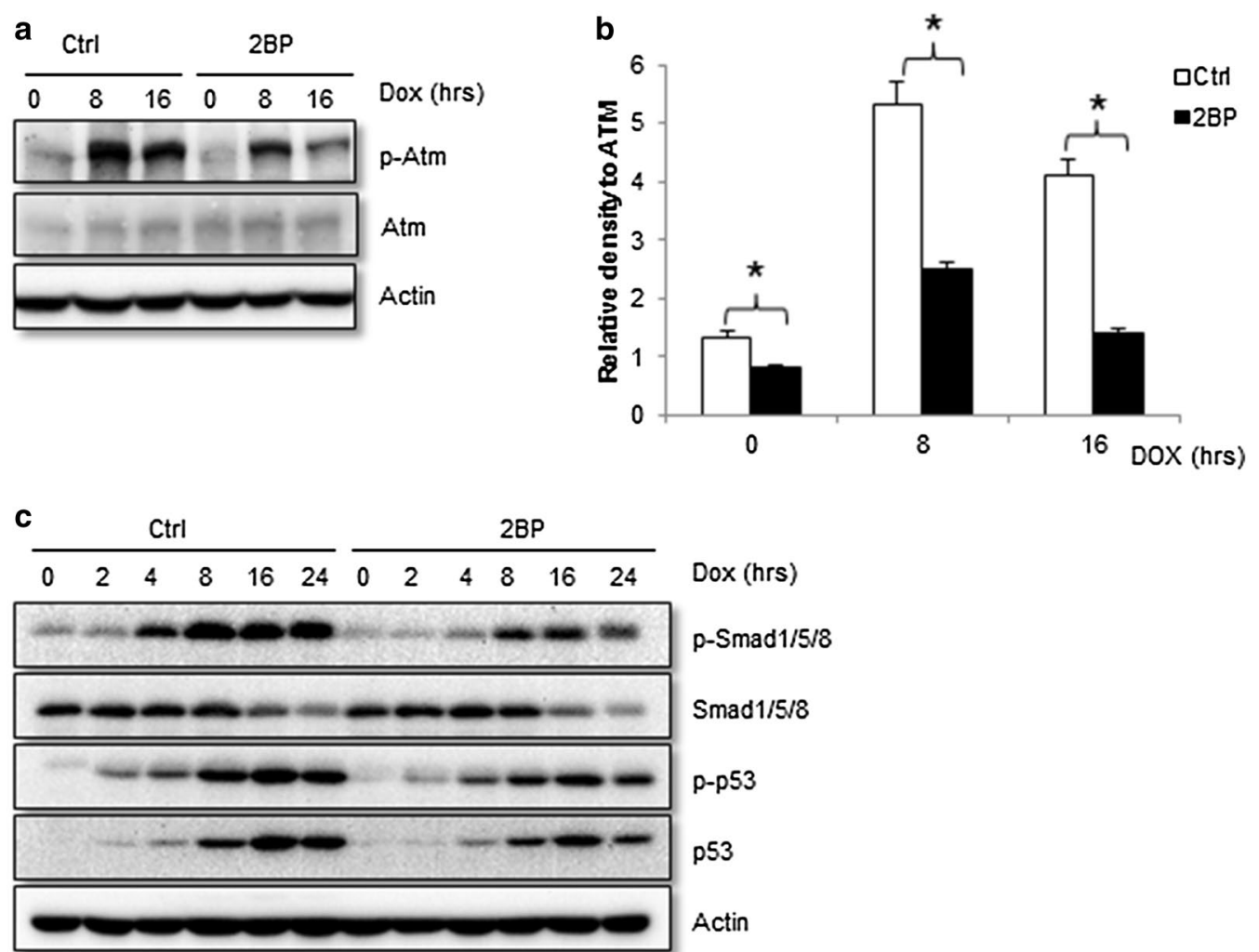

Fig. 3 Inhibition of palmitoylation impaired DNA damage-induced Atm activation. a Western blot analysis showed that inhibition of palmitoyltransferase by 2BP led to a decrease in Atm phosphorylation on S1981. Primary MEFs were pre-treated with $50 \mu \mathrm{M} 2 \mathrm{BP}$ for $24 \mathrm{~h}$ followed by $1 \mu \mathrm{M}$ Dox for different periods of time. b Quantitation of phosphorylated Atm at Ser1981 after being normalized to total Atm. Asterisk denoted significant difference $(p<0.05)$ between control and 2BP treated cells. c 2BP compromised DNA damage-induced Smad1 activation. Cells were treated the same as described in $3 \mathrm{~A}$ and probed for total and phosphorylated Smad1/5/8 and p53

zDHHC16 and other PATs likely regulate the protein(s) that affect Atm activation upon DNA damage. Atm activation requires MRN complex (a protein complex consisting of Mre11, Rad50 and Nbs1), DNA conformational change, and/or DNA breaks. The generally agreed major function of palmitoylation is to increase the hydrophobicity of the targeted protein, thus facilitate protein anchoring to membrane and subsequent interaction with other proteins $[1,2,13]$. Since most of the DNA damage foci proteins and signaling molecules are localized in the nucleus, it is more likely that palmitoylation affects the stability and/or complex assembly of the proteins that are involved in DNA damage response rather than their membrane association. One such candidate may be histone protein, which has been reported to be palmitoylated $[39,40]$. Palmitoylation of histone proteins may affect the remodeling of chromatin structures, which may in turn affect DNA damage foci formation and/or
DNA conformation, eventually lead to alteration of Atm activation and DNA repair [21, 41]. Whether histone proteins are substrates of zDHHC16 warrants further investigation.

Alternatively, zDHHC16 protein may regulate DNA damage response through c-Abl. zDHHC16 was originally identified as a c-Abl interacting protein, which was also named Aph2 [34]. c-Abl is activated in Atm-dependent manner in response to DNA damage. Activated c-Abl helps to up-regulate p53 and p73 expression and also plays a positive role in maximal Atm/Atr activation [3537]. $\mathrm{zDHHC16}$ seems to have a similar role as c-Abl in Atm activation in DNA damage response, yet c-Abl is not a substrate of $\mathrm{zDHHC16}$ [12]. It is possible that $\mathrm{zDHHC16}$ may regulate $\mathrm{DDR}$ via $\mathrm{c}-\mathrm{Abl}$ in a palmitoylation-independent manner. Further exploration on the nature of interaction between $\mathrm{zDHHC16}$ and c-Abl will help understand how zDHHC16 affects DNA damage response. 


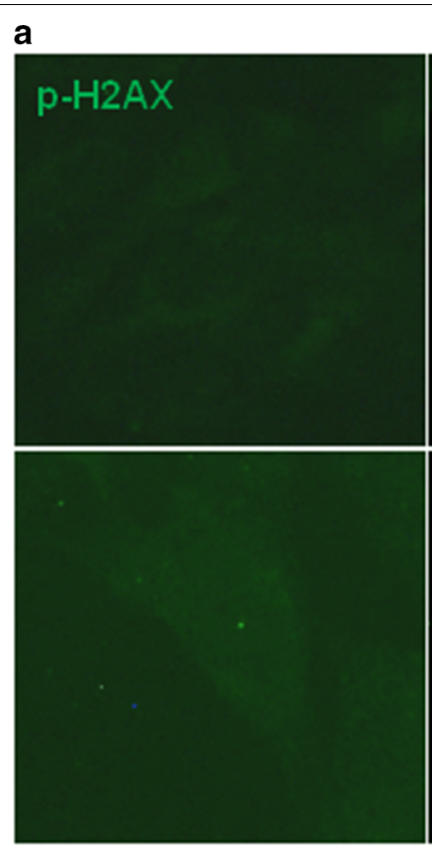

Dox $0 \mathrm{hr}$

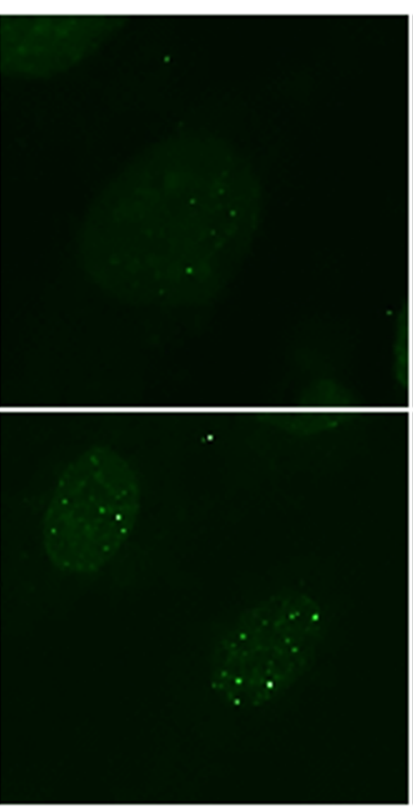

Dox $8 \mathrm{hrs}$

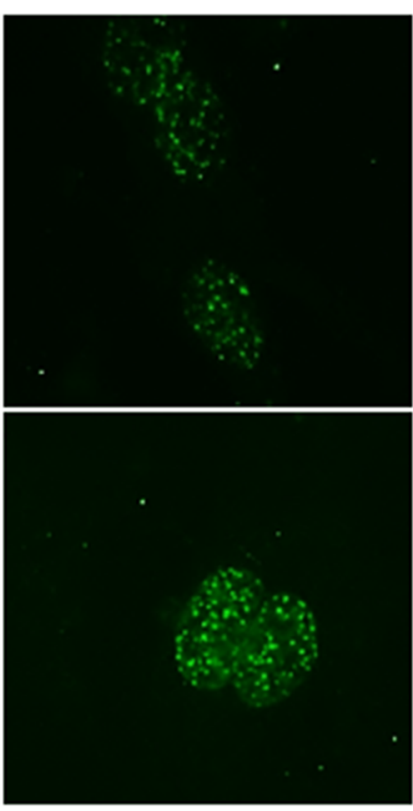

Dox16hrs

b

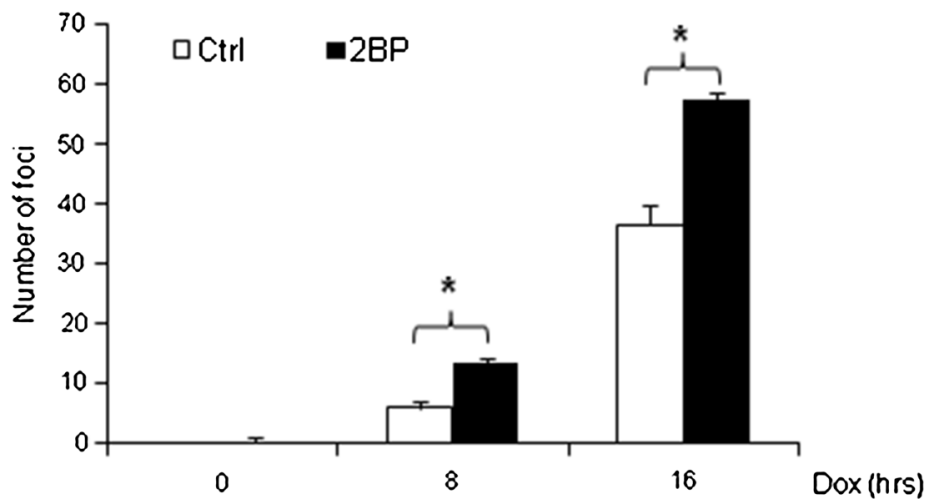

Fig. 4 Inhibition of palmitoylation impaired assembly/disassembly of DNA damage foci. Primary MEFs grown on coverslips were pre-treated with $50 \mu \mathrm{M} 2 \mathrm{BP}$ for $24 \mathrm{~h}$ and then treated with $1 \mu \mathrm{M}$ Dox for different periods of time. The cells were then fixed and immune-stained for H2AX using Texas-red conjugated secondary antibodies (a). b Averaged number of foci per cell from multiple repeated experiments and multiple cells per experiment. Asterisk denoted significant difference $(p<0.05)$ between compared groups

\section{Conclusions}

In summary, this study, for the first time, uncovered a critical role for protein palmitoylation and more specifically zDHHC16 in DNA damage response. These findings advance our understanding of the regulation of
DNA damage response and provide a possible explanation on how protein palmitoylation is involved in cancer development. Moreover, our results expand the role of palmitoylation on cellular activities and calls for further research on palmitoylated nuclear proteins. 

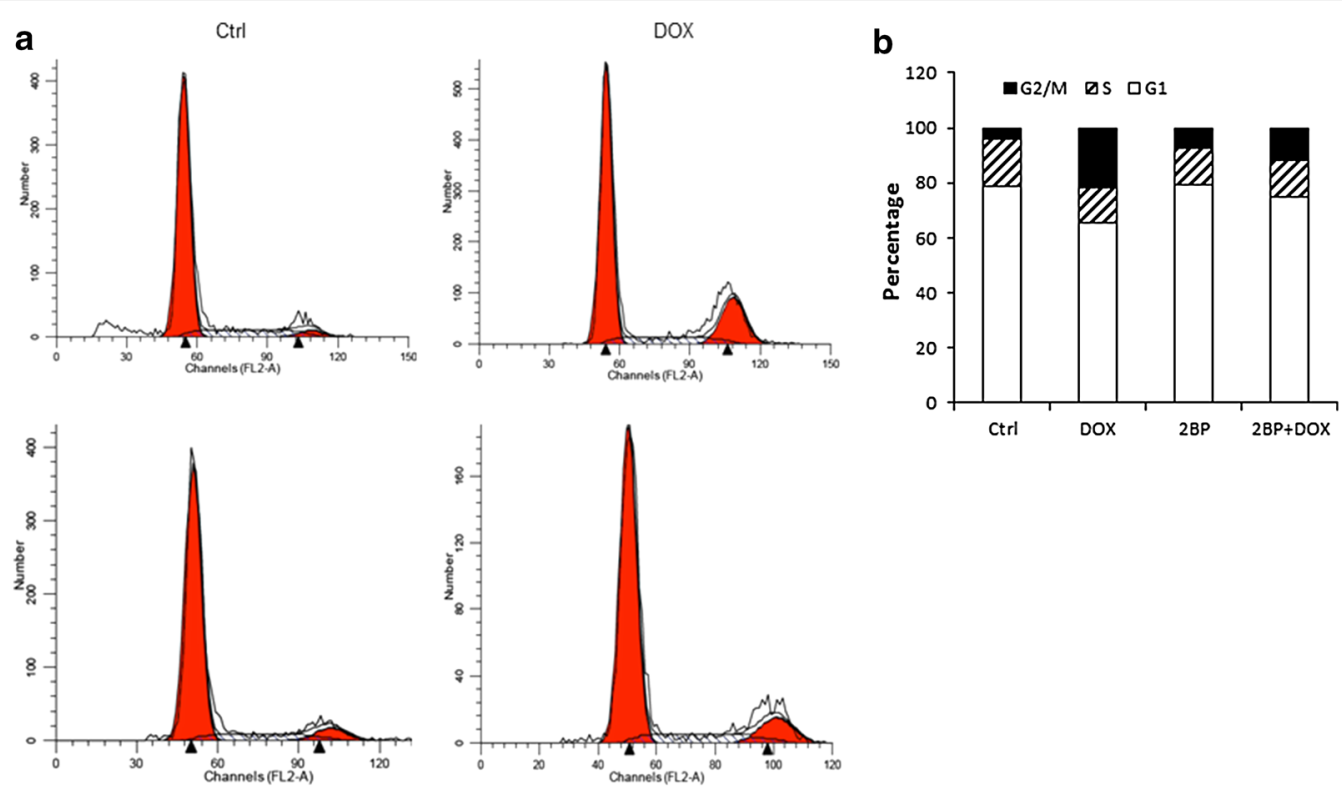

\section{$2 \mathrm{BP}+\mathrm{DOX}$}

Fig. 5 Inhibition of palmitoylation impaired DNA damage-induced cell cycle arrest at G2/M phase. Primary MEFs were pre-treated with $50 \mu M$ 2BP for $24 \mathrm{~h}$ and then treated with $1 \mu \mathrm{M}$ of Dox for $24 \mathrm{~h}$. The cells were harvested, fixed, stained with Pl, and analyzed by flow cytometry. a Representative micrographs of flow cytometry analysis. b Averaged data from multiple experiments. The total number of cells analyzed was set as $100 \%$ and cells at different phase were calculated accordingly

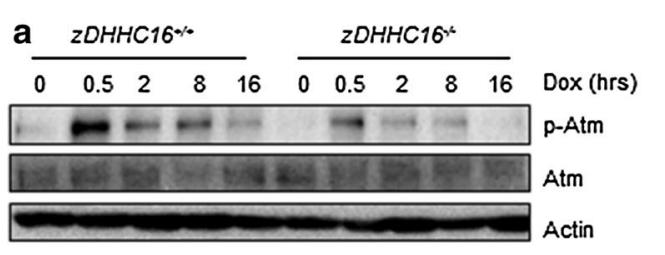

C $\quad z D H H C 16^{\%}$

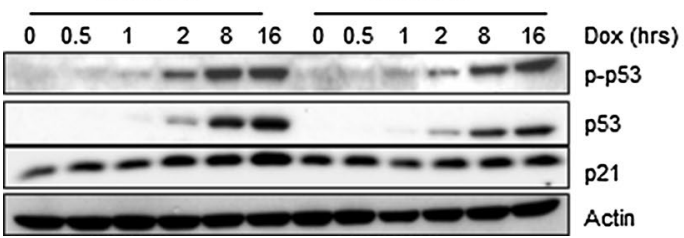

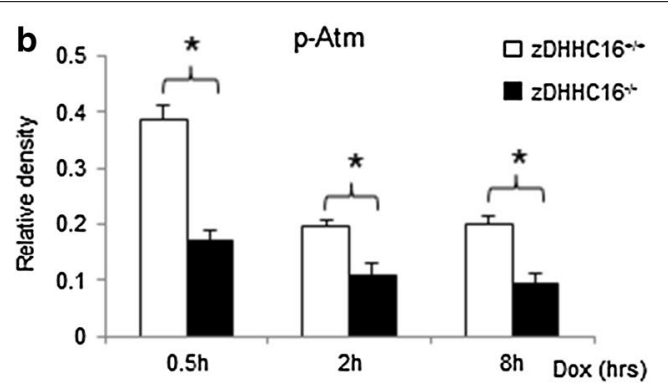

Fig. 6 zDHHC16 deficiency impaired DNA damage-induced Atm-p53 activation. a Western blot analysis showed reduced Atm phosphorylation at

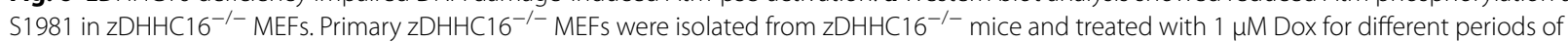
time as indicated. $\mathbf{b}$ Quantitation data on $\mathrm{p}$-Atm. Asterisk denoted significant difference $(p<0.05)$ between control and 2BP treated cells. c Activation and induction of p53 and p21. Cells were treated the same as above 

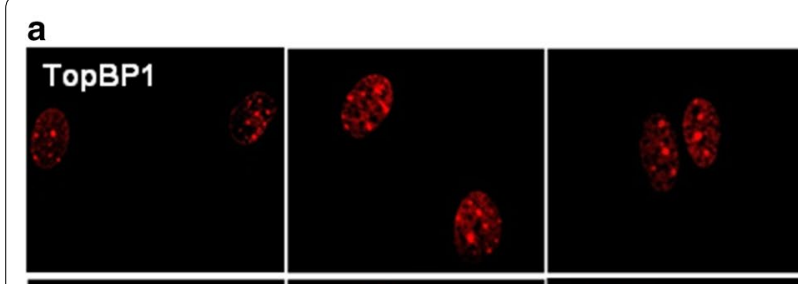

$2 \mathrm{OHHC} 16^{+/ 4}$
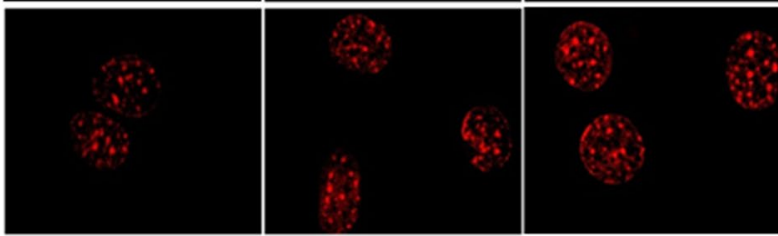

Dox 2hrs

Dox Ehrs

Dox16hrs

c

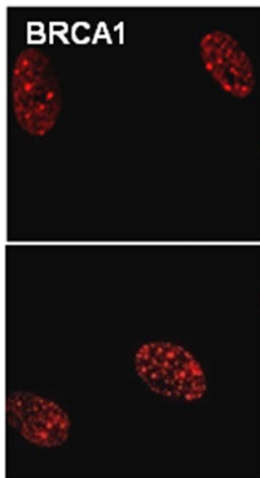

Dox 2hrs

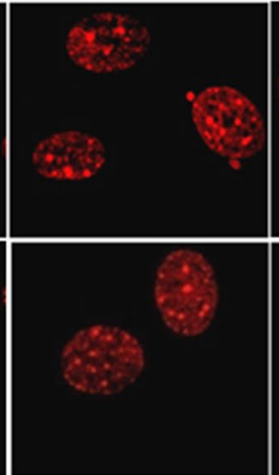

DoxGhrs

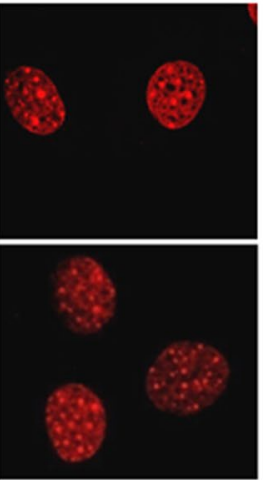

Dox16hrs

$2 \mathrm{DHHC} 16^{+/+}$

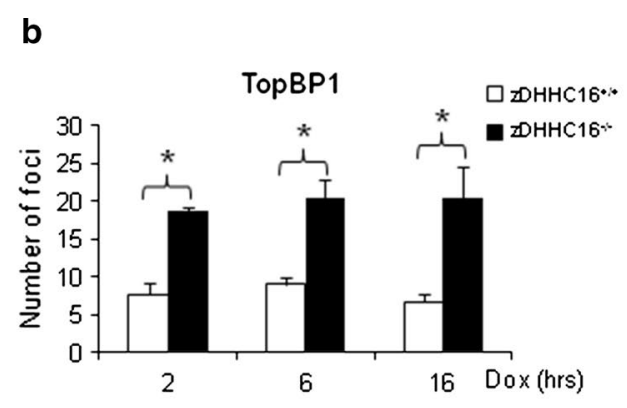

$2 \mathrm{DHHC16} 6^{--}$
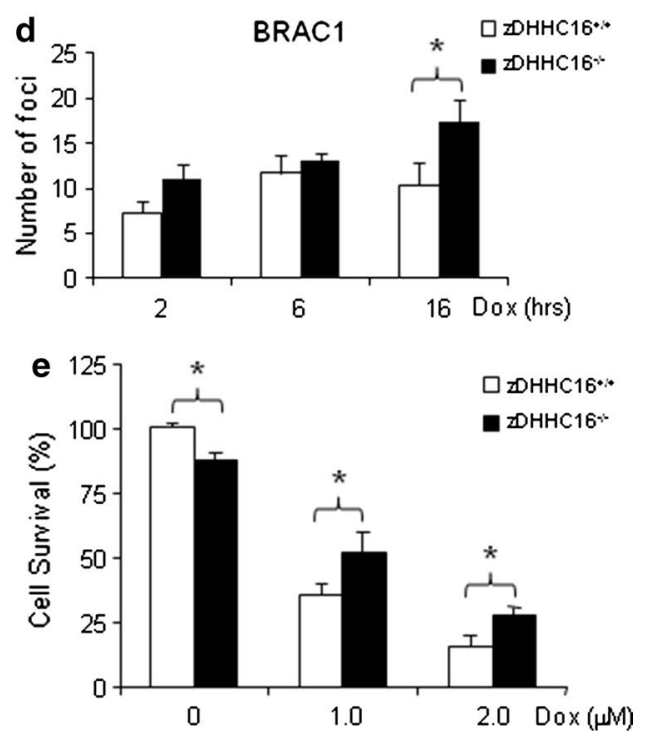

Fig. 7 zDHHC16 deficiency impaired the assembly/disassembly of DNA damage foci. Wild type and zDHHC16 ${ }^{-/-}$MEFs grown on coverslips were treated with $1 \mu$ M Dox for different periods of time. The cells were then fixed and immuno-stained for TopBP1 (a) and BRCA1 (c) using Texas-red conjugated secondary antibodies. The averaged numbers of foci under each condition were counted and averaged and presented at panels $\mathbf{b}$ and d, respectively. e $\mathrm{ZDHHC16}$ deficiency resulted in an increase in cell survival in response to Dox treatment. WT and zDHHC16 $6^{-1-} \mathrm{MEFs}$ were treated with 1 or $2 \mu \mathrm{M}$ Dox for $24 \mathrm{~h}$. Cell survival rates were determined by the Wst-1 assay. Asterisk denoted significant difference between wild type and $\mathrm{zDHHC} 16^{-/-} \mathrm{MEFs}$

\section{Additional file}

Additional file 1: Figure S1. 2BP inhibited protein palmitoylation in MEFs. Figure S2. The effect of 2BP on zDHHC gene expression in MEFs. Figure S3. The effect of $2 \mathrm{BP}$ and Dox on MEF cell survival. Figure S4. Overexpressed zDHHC16 increased protein palmitoylation in MEFs and was translocated into the nucleus in response to Dox.

\section{Abbreviations}

zDHHC: zinc-finger and Asp-His-His-Cys domains; PAT: protein acyltransferase; DDR: DNA damage response; PIKKs: phosphatidylinositol-3 kinase-related kinases; MEF: mouse embryonic fibroblast; 2BP: 2-bromopalmitate; DOX: doxorubicin; PI: propidium iodide; GAPDH: glyceraldehyde 3-phosphate; RT: reverse transcription; PCR: polymerase chain reaction.

\section{Authors' contributions}

$J \mathrm{~L}, \mathrm{LZ}$ and $\mathrm{BL}$ designed the research, analyzed the data and wrote the paper. NC and LZ did the western blot, immunofluorescent staining and FACS analysis; JKL and YQR performed the RT, real-time PCR and cytotoxicity analysis, HL isolated primary mouse embryonic fibroblasts; $\mathrm{PZ}$ and JW provided critical review on the whole project. All authors have read and approved the final manuscript.

\section{Author details}

${ }^{1}$ Bio-X Institutes, Key Laboratory for the Genetics of Developmental and Neuropsychiatric Disorders, Ministry of Education, Shanghai Jiao Tong University, Shanghai 200240, China. ${ }^{2}$ Department of Ophthalmology, Xin Hua Hospital, Shanghai Jiao Tong University School of Medicine, Shanghai, China. ${ }^{3}$ Neural Stem Cell Research Lab, Research Department, National Neuroscience Institute, Singapore 308433, Singapore.

\section{Acknowledgements}

We would like to thank Lina Gao and Haiyang Xie for their technical support.

\section{Availability of data and material}

The original data of the real-time PCR experiments and data images for the microscopy work and western blot analysis will be available upon request.

\section{Competing interests}

The authors declare that they have no competing interests. 


\section{Ethics approva}

The study was approved by the Institutional Animal Care and Use Committee of Shanghai Jiao Tong University [YXK(SH)2011-0112].

\section{Funding}

The work was supported by grants from the National Key Basic Research Program of China (2012CB966901 and 2014CB942902 to B. L.), which supported the design of the study, payment for the manpower and animal husbandry; the Technology Commission of Shanghai Municipality, China (12140903500 to J. L.) which supported the implementation of the experiments; and the National Natural Science Foundation of China (81371063 to J. L. and 81130039 to B. L.) which supported the data interpretation and the manuscript preparation.

Received: 1 January 2016 Accepted: 15 April 2016

Published online: 10 May 2016

\section{References}

1. Resh MD. Palmitoylation of ligands, receptors, and intracellular signaling molecules. Sci STKE. 2006;359:re14

2. Linder ME, Deschenes RJ. Palmitoylation: policing protein stability and traffic. Nat Rev Mol Cell Biol. 2007:8:74-84.

3. Blaskovic S, Adibekian A, Blanc M, van der Goot GF. Mechanistic effects of protein palmitoylation and the cellular consequences thereof. Chem Phys Lipids. 2014;180:44-52.

4. Korycka J, Lach A, Heger E, Boguslawska DM, Wolny M, Toporkiewicz M, Augoff K, Korzeniewski J, Sikorski AF. Human DHHC proteins: a spotlight on the hidden player of palmitoylation. Eur J Cell Biol. 2012;91:107-17.

5. Roth AF, Wan J, Bailey AO, Sun B, Kuchar JA, Green WN, Phinney BS, Yates JR 3rd, Davis NG. Global analysis of protein palmitoylation in yeast. Cell. 2006:125:1003-13.

6. Martin BR, Wang C, Adibekian A, Tully SE, Cravatt BF. Global profiling of dynamic protein palmitoylation. Nat Methods. 2012;9:84-9.

7. Adams MN, Christensen ME, He Y, Waterhouse NJ, Hooper JD. The role of palmitoylation in signalling, cellular trafficking and plasma membrane localization of protease-activated receptor-2. PLoS ONE. 2011;6:e28018.

8. Yang W, Di Vizio D, Kirchner M, Steen H, Freeman MR. Proteome scale characterization of human S-acylated proteins in lipid raft-enriched and non-raft membranes. Mol Cell Proteom. 2010;9:54-70.

9. Mukai J, Liu H, Burt RA, Swor DE, Lai WS, Karayiorgou M, Gogos JA Evidence that the gene encoding ZDHHC8 contributes to the risk of schizophrenia. Nat Genet. 2004:36:725-31.

10. Saleem AN, Chen YH, Baek HJ, Hsiao YW, Huang HW, Kao HJ, Liu KM, Shen LF, Song IW, Tu CP, et al. Mice with alopecia, osteoporosis, and systemic amyloidosis due to mutation in Zdhhc13, a gene coding for palmitoyl acyltransferase. PLoS Genet. 2010;6:e1000985.

11. Mill P, Lee AW, Fukata Y, Tsutsumi R, Fukata M, Keighren M, Porter RM, McKie L, Smyth I, Jackson IJ. Palmitoylation regulates epidermal homeostasis and hair follicle differentiation. PLoS Genet. 2009;5:e1000748.

12. Zhou T, Li J, Zhao P, Liu H, Jia D, Jia H, He L, Cang Y, Boast S, Chen YH, et al. Palmitoyl acyltransferase Aph2 in cardiac function and the development of cardiomyopathy. Proc Natl Acad Sci USA. 2015;112:15666-71.

13. Yeste-Velasco M, Linder ME, Lu YJ. Protein S-palmitoylation and cancer. Biochim Biophys Acta. 2015;1856:107-20.

14. Greaves J, Chamberlain LH. DHHC palmitoyl transferases: substrate interactions and (patho) physiology. Trends Biochem Sci. 2011;36:245-53.

15. Hanahan D, Weinberg RA. Hallmarks of cancer: the next generation. Cell. 2011;144:646-74.

16. Elledge SJ. The DNA damage response-self-awareness for DNA: the 2015 Albert Lasker Basic Medical Research Award. JAMA. 2015;314:1111-2.

17. Haber JE. Deciphering the DNA damage response. Cell. 2015;162:1183-5.

18. Jackson SP, Bartek J. The DNA-damage response in human biology and disease. Nature. 2009;461:1071-8.

19. Rothkamm K, Barnard S, Moquet J, Ellender M, Rana Z, Burdak-Rothkamm S. DNA damage foci: meaning and significance. Environ Mol Mutagen. 2015:56:491-504.
20. Rogakou EP, Boon C, Redon C, Bonner WM. Megabase chromatin domains involved in DNA double-strand breaks in vivo. J Cell Biol. 1999;146:905-16.

21. Wu J, Clingen PH, Spanswick VJ, Mellinas-Gomez M, Meyer T, Puzanov I, Jodrell D, Hochhauser D, Hartley JA. Gamma-H2AX foci formation as a pharmacodynamic marker of DNA damage produced by DNA crosslinking agents: results from 2 phase I clinical trials of SJG-136 (SG2000). Clin Cancer Res. 2013;19:721-30.

22. Paull TT. Mechanisms of ATM Activation. Annu Rev Biochem. 2015;84:711-38.

23. Shiloh Y Z Ziv Y. The ATM protein kinase: regulating the cellular response to genotoxic stress, and more. Nat Rev Mol Cell Biol. 2013;14:197-210.

24. Chau JF, Jia D, Wang Z, Liu Z, Hu Y, Zhang X, Jia H, Lai KP, Leong WF, Au BJ, et al. A crucial role for bone morphogenetic protein-Smad1 signalling in the DNA damage response. Nat Commun. 2012;3:836.

25. Ruan X, Zuo Q, Jia H, Chau J, Lin J, Ao J, Xia X, Liu H, Habib SL, Fu C, et al. P53 deficiency-induced Smad 1 upregulation suppresses tumorigenesis and causes chemoresistance in colorectal cancers. J Mol Cell Biol. 2015;7:105-18

26. Davda D, El Azzouny MA, Tom CT, Hernandez JL, Majmudar JD, Kennedy RT, Martin BR. Profiling targets of the irreversible palmitoylation inhibitor 2-bromopalmitate. ACS Chem Biol. 2013:8:1912-7.

27. Jennings $B C$, Nadolski MJ, Ling Y, Baker MB, Harrison ML, Deschenes RJ, Linder ME. 2-Bromopalmitate and 2-(2-hydroxy-5-nitro-benzylidene)benzo[b]thiophen-3-one inhibit DHHC-mediated palmitoylation in vitro. J Lipid Res. 2009;50:233-42.

28. Resh MD. Use of analogs and inhibitors to study the functional significance of protein palmitoylation. Methods. 2006:40:191-7.

29. Carvalho C, Santos RX, Cardoso S, Correia S, Oliveira PJ, Santos MS, Moreira PI. Doxorubicin: the good, the bad and the ugly effect. Curr Med Chem. 2009;16:3267-85.

30. Garant KA, Shmulevitz M, Pan L, Daigle RM, Ahn DG, Gujar SA, Lee PW. Oncolytic reovirus induces intracellular redistribution of Ras to promote apoptosis and progeny virus release. Oncogene. 2015;35(6):771-82.

31. Fukata Y, Dimitrov A, Boncompain G, Vielemeyer O, Perez F, Fukata M. Local palmitoylation cycles define activity-regulated postsynaptic subdomains. J Cell Biol. 2013;202:145-61.

32. Zheng B, DeRan M, Li X, Liao X, Fukata M, Wu X. 2-bromopalmitate analogues as activity-based probes to explore palmitoyl acyltransferases. J Am Chem Soc. 2013;135:7082-5.

33. Leong WF, Zhou T, Lim GL, Li B. Protein palmitoylation regulates osteoblast differentiation through BMP-induced osterix expression. PLoS ONE. 2009; $4:$ e4135

34. Li B, Cong F, Tan CP, Wang SX, Goff SP. Aph2, a protein with a zf-DHHC motif, interacts with c-Abl and has pro-apoptotic activity. J Biol Chem. 2002;277:28870-6

35. Maiani E, Diederich M, Gonfloni S. DNA damage response: the emerging role of c-Abl as a regulatory switch? Biochem Pharmacol. 2011:82:1269-76.

36. Meltser $\mathrm{V}$, Ben-Yehoyada M, Shaul Y. c-Abl tyrosine kinase in the DNA damage response: cell death and more. Cell Death Diff. 2011;18:2-4.

37. Shaul Y, Ben-Yehoyada M. Role of c-Abl in the DNA damage stress response. Cell Res. 2005;15:33-5.

38. Wang X, Zeng L, Wang J, Chau JF, Lai KP, Jia D, Poonepalli A, Hande MP, Liu $\mathrm{H}, \mathrm{He} \mathrm{G}$, et al. A positive role for $\mathrm{C}-\mathrm{Abl}$ in Atm and Atr activation in DNA damage response. Cell Death Diff. 2011;18:5-15.

39. Chen X, Du Z, Shi W, Wang C, Yang Y, Wang F, Yao Y, He K, Hao A. 2-Bromopalmitate modulates neuronal differentiation through the regulation of histone acetylation. Stem Cell Res. 2014;12:481-91.

40. Wilson JP, Raghavan AS, Yang YY, Charron G, Hang HC. Proteomic analysis of fatty-acylated proteins in mammalian cells with chemical reporters reveals S-acylation of histone $\mathrm{H} 3$ variants. Mol Cell Proteom. 2011;10(M110):001198.

41. Hsiao KY, Mizzen CA. Histone H4 deacetylation facilitates 53BP1 DNA damage signaling and double-strand break repair. J Mol Cell Biol. 2013;5:157-65. 